\title{
MicroRNA-383-5p predicts favorable prognosis and inhibits the progression of diffuse large B-cell lymphoma
}

\author{
LI-YAN CHEN, BI-QING HAN, XIAO-MIN ZHANG, XING-BAO YU, DAN-DAN YAO and LI-QIAN YU \\ Department of Hematology, The Second Affiliated Hospital of Harbin Medical University, \\ Harbin, Heilongjiang 150001, P.R. China
}

Received July 11, 2020; Accepted February 10, 2021

DOI: $10.3892 / 01.2021 .12776$

\begin{abstract}
The roles of microRNA (miRNA/miR)-383-5p have been reported in several malignancies, including breast cancer, gastric cancer, ovarian cancer and lung adenocarcinoma. However, its function in diffuse large B-cell lymphoma (DLBCL) remains unclear. Thus, the present study aimed to investigate the role of miR-383-5p in DLCBL. Reverse transcription-quantitative PCR analysis was performed to detect miR-383-5p expression in 80 paired tissue samples from patients with DLBCL and control subjects, as well as related cancer cell lines. Kaplan-Meier survival analysis was performed, and the prognostic value of miR-383-5p was determined via Cox regression analysis. Furthermore, the association between miR-383-5p expression and the clinicopathological characteristics of patients with DLBCL was investigated. The Cell Counting Kit-8, crystal violet staining and Transwell assays were performed to assess the effects of miR-383-5p on cell proliferation and invasion, respectively. The results demonstrated that miR-383-5p expression was upregulated in human DLBCL tissues and cell lines. In addition, miR-383-5p expression was closely associated with clinical stage and extranodal invasion in patients with DLBCL. Notably, high miR-383-5p expression was able to predict a favorable clinical prognosis in patients with DLBCL. Furthermore, overexpression of miR-383-5p significantly inhibited the proliferation and invasion of DLBCL cells, the effects of which were reversed following miR-383-5p knockdown. Taken together, the results of the present study suggest that miR-383-5p may predict favorable prognosis, and thus may be used as a prognostic biomarker for patients with DLBCL. In addition, miR-383-5p appears to play critical roles in inhibiting the proliferation and invasion of DLBCL cells, and thus may be used as a potential therapeutic target in patients with DLBCL.
\end{abstract}

Correspondence to: Dr Li-Yan Chen, Department of Hematology, The Second Affiliated Hospital of Harbin Medical University, 246 Xuefu Road, Nangang, Harbin, Heilongjiang 150001, P.R. China E-mail: freetime567@163.com

Key words: microRNA-383-5p, diffuse large B-cell lymphoma, prognosis, cancer progression

\section{Introduction}

Lymphoma is one of the most common cancers worldwide, with an incidence approximately 6.68 per 100,000 people, and develops in hematological and lymphoid compartments, including lymph nodes, spleen and thymus, as well as extranodal lymphatic tissues and organs $(1,2)$. Diffuse large B-cell lymphoma (DLBCL) accounts for $\sim 30.0 \%$ of non-Hodgkin's lymphoma in western countries and $45.8 \%$ in China (3). The incidence of DLBCL continues to increase $(4,5)$. Notably, DLBCL is a clinically, pathologically and molecularly heterogeneous entity, with a 5-year survival rate of 32-81\% (6). Despite improvements in its response rate to rituximab, and combination with chemotherapy and other novel treatment modalities, such as promising immunotherapy, a significant proportion of patients with DLBCL exhibit strong refractory and relapse trends, thus the long-term prognosis of this disease remains unsatisfactory (7). Thus, identification of novel prognostic biomarkers and therapeutic targets for effective diagnosis and treatment of patients with DLBCL is of great importance.

MicroRNAs (miRNAs/miRs) are small non-coding RNAs, 20-25 nucleotides in length (8). Recently, several studies have demonstrated that miRNAs are involved in an extensive range of biological processes, including cell proliferation, migration, invasion, and apoptosis (9-11). In addition, some miRNAs act as oncogenes or tumor suppressor genes in different types of cancer (12-14). miR-383-5p is located at chromosome $8 \mathrm{p} 22$ in humans (15), and its role as a tumor suppressor has been investigated in several malignancies, including breast (15), gastric $(16,17)$ and ovarian cancers $(18)$, as well as lung adenocarcinoma (19) and oral squamous cell carcinoma (20). With regards to DLBCL, several studies have reported that some miRNA signatures are closely associated with characteristics of patients with DLBCL, including cell proliferation, migration, invasion and chemotherapy-resistance, as well as clinical practice (21-23). However, the biological function and prognostic value of miR-383-5p in DLBCL remains unclear.

The present study aimed to investigate the biological functions and determine the prognostic value of miR-383-5p in DLBCL progression. The expression pattern of miR-383-5p, as well as its association with clinicopathological characteristics of patients with DLBCL were assessed. The in vitro effects of miR-383-5p on the proliferation and invasion of DLBCL cells was also investigated. 


\section{Materials and methods}

Patient samples and clinical follow-up. A total of 80 patients with DLBCL at the Second Affiliated Hospital of Harbin Medical University (Harbin, China) were enrolled in the present study between January 2012 and December 2016. The inclusion criteria were as follows: Patients with DLBCL underwent routine $\mathrm{R}-\mathrm{CHOP}$ and received no previous chemotherapy or radiation. Patients with incomplete follow-up data, including clinicopathological characteristics and survival time were excluded from the present study.

DLBCL tissue samples were collected via biopsy and stored at $-80^{\circ} \mathrm{C}$ until subsequent experimentation. Control biopsy tissue samples $(n=80)$ were collected from patients with reactive lymphoid hyperplasia (RLH, suspected of lymphoma). The pathological status of all samples was independently confirmed by two pathologists at the Second Affiliated Hospital of Harbin Medical University. Patients with DLBCL were assessed once every 4-6 months, until death or dropout from the telephone follow-up program. The follow-up program was between January 2012 and December 2019. Clinical parameters and survival information were recorded accordingly. The clinical parameters included age, sex, B symptoms, clinical stage (24), extranodal invasion, serum lactate dehydrogenase (LDH) levels and International Prognostic Index (IPI) score (25). Patient follow-up was censored on December 2019. The present study was approved by the Ethics Committee of the Second Affiliated Hospital of Harbin Medical University (Harbin, China; approval no. ASHHM000152), and performed in accordance with the Declaration of Helsinki. Written informed consent was provided by all patients prior to the study start.

Classification of patients. Patients were divided into two groups (miR-383-5p low and high expression groups), based on median miR-383-5p expression or the 25 th percentile miR-383-5p expression. Overall survival (OS) was defined from the time of treatment to death or last follow-up, while disease-free survival (DFS) was defined from the time of treatment to recurrence, death or last follow-up.

Reverse transcription-quantitative $(R T-q) P C R$. Total miRNA was extracted from fresh tissues using the RNA Extraction kit (Qiagen, Inc.), according to the manufacturer's protocol, and reverse transcribed into cDNA using the RT kit (Shanghai GenePharma Co., Ltd.). The temperature protocol for RT was $51^{\circ} \mathrm{C}$ for $20 \mathrm{~min}$, followed by $82^{\circ} \mathrm{C}$ for $10 \mathrm{~min}$. A miR-383-5p-specific primer and probe (TaqMan MicroRNA Assay kit, Applied Biosystems; Thermo Fisher Scientific, Inc.) were used to detect expression levels, using an ABI 7500 FAST Real-Time PCR system (Applied Biosystems; Thermo Fisher Scientific, Inc.). The following thermocycling conditions were used for qPCR: Initial denaturation at $94^{\circ} \mathrm{C}$ for $2 \mathrm{~min}$, followed by 40 cycles of $94^{\circ} \mathrm{C}$ for $10 \mathrm{sec}, 55^{\circ} \mathrm{C}$ for $30 \mathrm{sec}$ and $72^{\circ} \mathrm{C}$ for $20 \mathrm{sec}$. The following primer sequences were used for qPCR: miR-383-5p forward, 5'-GGGAGATCA GAAGGTGATTGTGGCT-3' and reverse, 5'-CAGTGCGTG TCGTGGAGT-3'; and U6 forward, 5'-CTCGCTTCGGCA GCACA-3' and reverse, 5'-AACGCTTCACGAATTTGC GT-3'. Relative miR-383-5p expression levels were calculated using the $2^{-\Delta \Delta \mathrm{Cq}}$ method (26) and normalized to the internal reference gene U6.

Cell culture and transfection. Human DLBCL cell lines, OCI-LY7 and OCI-LY3, and normal B lymphocytes, IM-9I, were purchased from the American Type Culture Collection. OCI-LY7 and OCI-LY3 cells were maintained in IMEM medium (Gibco; Thermo Fisher Scientific, Inc.) supplemented with $10 \%$ fetal bovine serum (FBS; Thermo Fisher Scientific, Inc.), while IM-9I cells were maintained in RPMI-1640 culture medium (Gibco; Thermo Fisher Scientific, Inc.) supplemented with $10 \%$ FBS. All cells were cultured at $37^{\circ} \mathrm{C}$ with $5 \% \mathrm{CO}_{2}$.

miR-383-5p mimic, miR-383-5p inhibitor and corresponding negative controls (NC) were purchased from Shanghai GenePharma Co., Ltd.. Once they reached $70 \%$ confluence, $2 \times 10^{5}$ cells were transfected with $50 \mathrm{nM}$ miR-383-5p mimic, miR-383-5p inhibitor and/or respective controls, using Lipofectamine ${ }^{\circledR} 2000$ reagent (Invitrogen; Thermo Fisher Scientific, Inc.) at $37^{\circ} \mathrm{C}$ for $3 \mathrm{~h}$, according to the manufacturer's protocol. The following sequences were used: miR-383-5p mimic, 5'-AAAUCUCUGCAGGCAAAUGUG A-3' and mimic-NC, 5'-UUCUCCGAACGUGUCACGUTT-3'; and miR-383-5p inhibitor, 5'-CAGUGGUUUUACCCUAUG GUAG-3' and inhibitor-NC, 5'-UUCUCCGAACGUGUCACG UTT-3'. Cells were harvested for subsequent experimentation $48 \mathrm{~h}$ post-transfection.

Cell counting kit-8 (CCK-8) assay. Transfected cells were seeded into 96 -well plates at a density of $1 \times 10^{4}$ cells/well. CCK-8 reagent (10 $\mu$, Dojindo Molecular Technologies, Inc.) was added to each well at $0,24,48$, and $72 \mathrm{~h}$, and incubated at $37^{\circ} \mathrm{C}$ for an additional $2 \mathrm{~h}$. Cell proliferation was subsequently analyzed at a wavelength of $450 \mathrm{~nm}$, using an automatic microplate reader (BioTek Instruments).

Crystal violet staining. Crystal violet staining was performed to assess cell proliferation. Transfected cells were seeded into 6 -well plates at the density of 1,000 cells/well and cultured in IMEM medium (Gibco; Thermo Fisher Scientific, Inc.) supplemented with $10 \% \mathrm{FBS}$ at $37^{\circ} \mathrm{C}$. Culture medium was replaced every 3 days for a total of 2 weeks. Cells were subsequently fixed with $100 \%$ methanol for $5 \mathrm{~min}$ at room temperature and stained with $0.5 \%$ crystal violet solution for $10 \mathrm{~min}$ at room temperature. Cells were washed three times with PBS and observed under a light microscope (magnification, x4). Cell proliferation was subsequently analyzed at a wavelength of $570 \mathrm{~nm}$, using an automatic microplate reader.

Transwell assay. The Transwell assay was performed to assess cell invasion. A total of $100 \mu \mathrm{l}$ cell suspension without FBS $\left(4 \times 10^{4}\right.$ cells) was plated in the upper chambers of 24-well Transwell plates (Corning, Inc.), with $8 \mu \mathrm{m}$ pore size membranes. IMEM medium (Gibco; Thermo Fisher Scientific, Inc.) $(500 \mu \mathrm{l})$ supplemented with $10 \%$ FBS was plated in the lower chambers. Membranes were pre-coated with $50 \mu \mathrm{l}$ Matrigel (BD Biosciences) for $4 \mathrm{~h}$ at $37^{\circ} \mathrm{C}$. Following incubation for $24 \mathrm{~h}$ at $37^{\circ} \mathrm{C}$ with $5 \% \mathrm{CO}_{2}$, the invasive cells were fixed with $100 \%$ methanol for $5 \mathrm{~min}$ at room temperature and stained with $0.1 \%$ crystal violet for $10 \mathrm{~min}$ at room temperature. Stained cells were counted in five randomly selected 

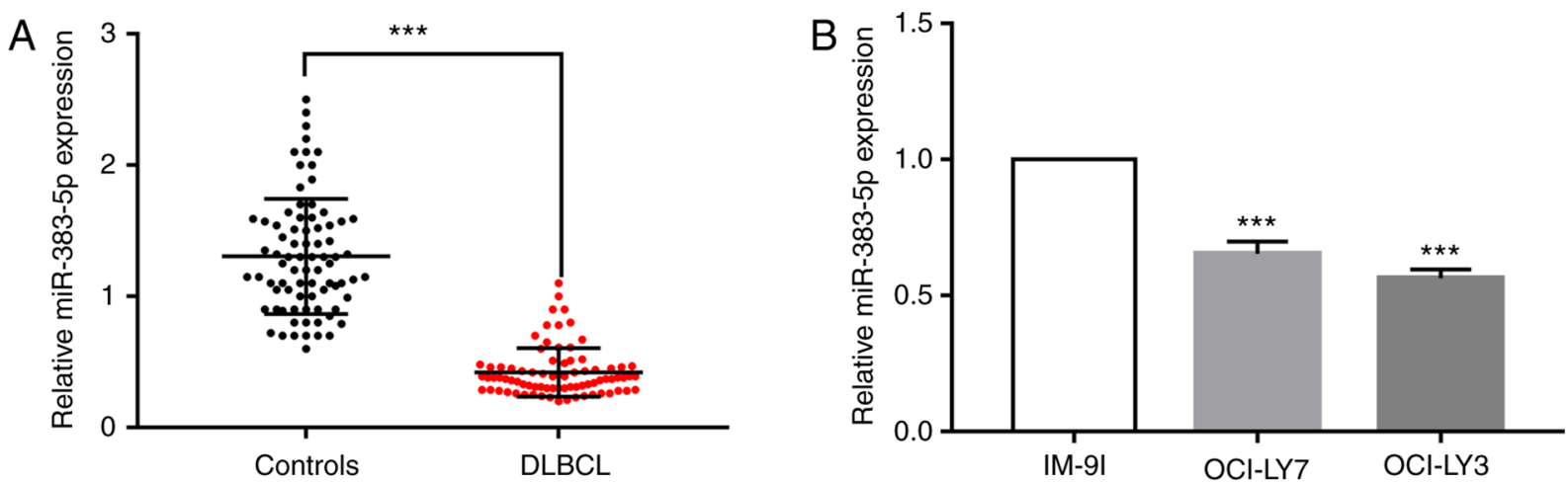

Figure 1. miR-383-5p expression is downregulated in DLBCL tissues and cell lines. (A) RT-qPCR analysis was performed to detect miR-383-5p expression in DLBCL tissues and control lymphoid hyperplasia tissues. (B) RT-qPCR analysis was performed to detect miR-383-5p expression in DLBCL cell lines (OCI-LY7 and OCI-LY3 cells) and normal B lymphocyte (IM-9I cells). Data are presented as the mean \pm standard deviation $(\mathrm{n}=3) .{ }^{* * *} \mathrm{P}<0.001 . \mathrm{miR}$, microRNA; DLBCL, diffuse large B-cell lymphoma; RT-qPCR, reverse transcription-quantitative PCR.

fields using a light microscope (magnification, x200; Olympus Corporation).

Statistical analysis. Statistical analysis was performed using GraphPad Prism 5.0 software (GraphPad Software Inc.) and SPSS 20.0 software (IBM Corp.). All experiments were performed in triplicate and data are presented as the mean \pm standard deviation. The $\chi^{2}$ test was used to assess the association between miR-383-5p expression and the clinicopathological characteristics of patients with DLBCL. Unpaired Student's t-test was used to compare differences between two groups, while one-way ANOVA followed by LSD test were used to compare differences between multiple groups. Kaplan-Meier curves were generated for both OS and DFS. Cox regression analysis was performed to determine the prognostic factors. $\mathrm{P}<0.05$ was considered to indicate a statistically significant difference.

\section{Results}

miR-383-5p expression is downregulated in DLBCL tissues and cell lines. RT-qPCR analysis was performed to detect miR-383-5p expression in 80 paired DLBCL tissues and normal tissues from patients with RLH. The results demonstrated that miR-383-5p expression was significantly downregulated in DLBCL tissues compared with RLH tissues $(\mathrm{P}<0.001$; Fig. 1A). miR-383-5p expression was also detected in the DLBCL cell lines (OCI-LY7 and OCI-LY3 cells) and normal B lymphocytes (IM-9I cells). As presented in Fig. 1B, miR-383-5p expression was significantly lower in DLBCL cells compared with IM-9I cells $(\mathrm{P}<0.001)$.

Patients with DLBCL were classified into two groups, miR-383-5p low expression $(n=40)$ and miR-383-5p high expression $(n=40)$ groups, based on median miR-383-5p expression.

Association between miR-383-5p expression and the clinicopathological characteristics of patients with DLBCL. The association between miR-383-5p expression and the clinicopathological characteristics of patients with DLBCL was assessed. As presented in Table I, patients with low miR-383-5p expression were significantly associated with advanced clinical stages of the disease $(\mathrm{P}=0.004)$. In addition, low miR-383-5p expression was significantly associated with a higher rate of extranodal invasion compared with the high miR-383-5p expression group ( $\mathrm{P}=0.001)$. Notably, no significant differences in age, sex, B symptoms, IPI score and serum LDH levels were observed between the two groups.

Kaplan-Meier survival analysis demonstrated that patients with high miR-383-5p expression had significantly higher OS $(\mathrm{P}=0.002$; Fig. 2A) and DFS ( $\mathrm{P}=0.04$; Fig. 2B) rates than those with low miR-383-5p expression. Univariate and multivariate Cox regression analyses were performed to determine the prognostic factors associated with OS and DFS.

The prognostic factors found to be significant in the univariate analysis $(\mathrm{P}<0.05)$ were subjected to multivariate analysis with the Cox proportional hazards regression model. Univariate and multivariate analyses indicated that OS was significantly associated with clinical stage [hazard ratio (HR), 1.570; 95\% confidence interval (CI), 1.402-1.759; $\mathrm{P}=0.001$ ], extranodal invasion (HR, 1.444; 95\% CI, 1.265-1.649; $\mathrm{P}=0.001$ ) and miR-383-5p expression (HR, 0.844; 95\% CI, 0.754-0.945; $\mathrm{P}=0.003$ ) (Table II). Similarly, multivariate analysis indicated that DFS was significantly associated with clinical stage (HR, 1.605; 95\% CI, 1.433-1.797; $\mathrm{P}<0.001$ ), extranodal invasion (HR, 1.734; 95\% CI, 1.520-1.981; $\mathrm{P}<0.001$ ) and miR-383-5p expression (HR, 0.753; 95\% CI, 0.672-0.842; $\mathrm{P}<0.001)$ (Table III).

Patients were classified into two groups, miR-383-5p high expression $(n=20)$ and miR-383-5p low expression $(n=60)$ groups, based on the 25th percentile. Kaplan-Meier survival analysis demonstrated that patients with high miR-383-5p expression had a significantly higher OS rate $(\mathrm{P}=0.032$; Fig. 2C). However, no significant difference in DFS rate was observed between the two groups ( $\mathrm{P}=0.217$; Fig. 2D). Taken together, these results suggest that miR-383-5p expression is downregulated in patients with DLBCL, and high miR-383-5p expression is associated with a favorable clinical prognosis.

Overexpression of miR-383-5p inhibits the proliferation and invasion of DLBCLcells. The biological function of miR-383-5p during DLBCL progression was investigated. OCI-LY7 and OCI-LY3 cells were transfected with miR-383-5p mimic, and the effect of overexpressing miR-383-5p on the proliferation 
Table I. Association between miR-383-5p expression and the clinicopathological characteristics of patients with diffuse large B-cell lymphoma $(\mathrm{n}=80)$.

miR-383-5p expression, $\mathrm{n}(\%)$

Characteristic

Patients, n

High $(\mathrm{n}=40)$

Low $(n=40)$

P-value

Age, years

$<60$

38

$18(45.0)$

$22(55.0)$

$\geq 60$

42

$14(35.0)$

Sex

Male

36

44

$26(65.0)$

B symptoms

Absent

36

$19(47.5)$

$21(52.5)$

Present

44

$28(70.0)$

$12(30.0)$

37

37

39

41

$27(57.5)$

13 (42.5)

Extranodal invasion

$<2$
$\geq 2$

34

$18(45.0)$

$22(55.0)$

46

38

42

Serum LDH level, IU/1

$\begin{array}{lllr}<300 & 38 & 23(57.5) & 15(37.5) \\ \geq 300 & 42 & 17(42.5) & 25(62.5)\end{array}$

$20(50.0)$

$20(50.0)$

$22(55.0)$

18 (45.0)

$17(42.5)$

$23(57.5)$

$15(37.5)$

25 (62.5)

0.654

0.072

$0.004^{\mathrm{a}}$

$0.001^{\mathrm{a}}$

$12(20.0)$

$28(80.0)$

$16(40.0)$

$24(60.0)$

0.073

${ }^{\text {a }}<<0.01$. miR, microRNA; IPI, International Prognostic Index; LDH, lactate dehydrogenase.

A

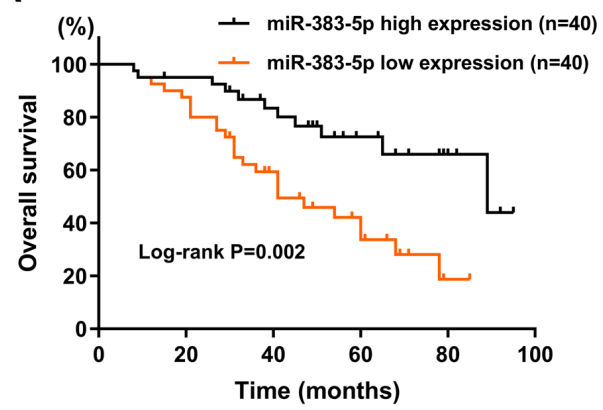

C

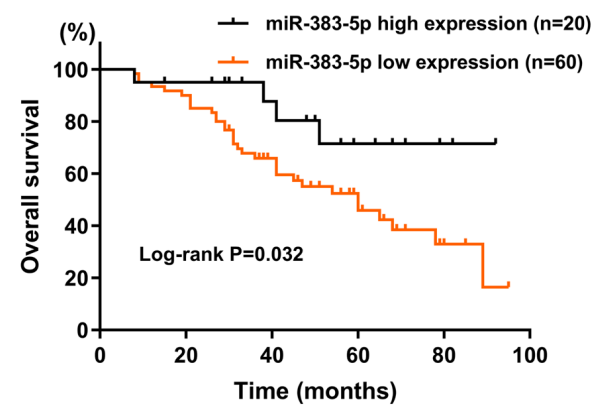

B

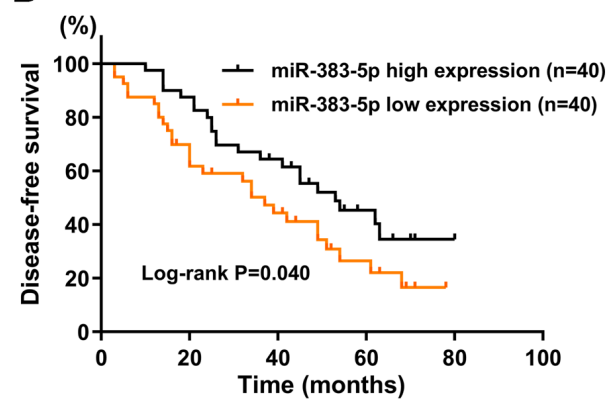

D

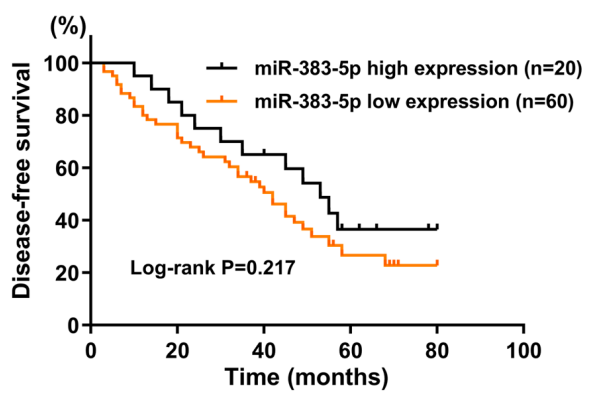

Figure 2. miR-383-5p expression is associated with the prognosis of patients with diffuse large B-cell lymphoma. Patients with high miR-383-5p expression had significantly higher (A) overall survival and (B) disease-free survival rates than those with low miR-383-5p expression. (C) Patients with high miR-383-5p expression had a significantly higher overall survival rate (D) but not disease-free survival rate than those with low miR-383-5p expression. miR, microRNA. 
Table II. Univariate and multivariate analyses of prognostic factors associated with overall survival.

\begin{tabular}{|c|c|c|c|c|}
\hline \multirow[b]{2}{*}{ Variable } & \multicolumn{2}{|c|}{ Univariate } & \multicolumn{2}{|c|}{ Multivariate } \\
\hline & HR $(95 \% \mathrm{CI})$ & $\mathrm{P}$-value & HR $(95 \%$ CI $)$ & P-value \\
\hline Age, years ( $\geq 60$ vs. $<60)$ & $1.005(0.841-1.200)$ & 0.931 & - & - \\
\hline Sex (Male vs. Female) & $1.036(0.830-1.292)$ & 0.755 & - & - \\
\hline B symptoms (Absent vs. Present) & $0.923(0.761-1.121)$ & 0.419 & - & - \\
\hline Clinical stage (I/II vs. III/IV) & $1.592(1.422-1.782)$ & $<0.001^{\mathrm{b}}$ & $1.570(1.402-1.759)$ & $0.001^{\mathrm{a}}$ \\
\hline Extranodal invasion $(\geq 2$ vs. $<2)$ & $1.448(1.268-1.656)$ & $<0.001^{\mathrm{b}}$ & $1.444(1.265-1.649)$ & $0.001^{\mathrm{a}}$ \\
\hline IPI score (3-5 vs. $0-2)$ & $1.231(0.686-2.423)$ & 0.376 & - & - \\
\hline Serum LDH level, IU/1 ( $\geq 300$ vs. $<300)$ & $1.983(0.881-3.123)$ & 0.556 & - & - \\
\hline miR-383-5p expression (High vs. Low) & $0.783(0.700-0.876)$ & $0.001^{\mathrm{a}}$ & $0.844(0.754-0.945)$ & $0.003^{\mathrm{a}}$ \\
\hline
\end{tabular}

${ }^{\mathrm{a}} \mathrm{P}<0.01,{ }^{\mathrm{b}} \mathrm{P}<0.001$. IPI, International Prognostic Index; LDH, lactate dehydrogenase; miR, microRNA; HR, hazard ratio; CI, confidence interval; -, not available.

Table III. Univariate and multivariate analyses of prognostic factors associated with disease-free survival.

\begin{tabular}{|c|c|c|c|c|}
\hline \multirow[b]{2}{*}{ Variable } & \multicolumn{2}{|c|}{ Univariate } & \multicolumn{2}{|c|}{ Multivariate } \\
\hline & $\mathrm{HR}(95 \% \mathrm{CI})$ & P-value & $\mathrm{HR}(95 \% \mathrm{CI})$ & $\mathrm{P}$-value \\
\hline Age, years ( $\geq 60$ vs. $<60)$ & $1.163(0.960-1.411)$ & 0.112 & - & - \\
\hline Sex (Male vs. Female) & $1.009(0.845-1.206)$ & 0.920 & - & - \\
\hline B symptoms (Absent vs. Present) & $1.006(0.878-1.152)$ & 0.931 & - & - \\
\hline Clinical stage (I/II vs. III/IV) & $1.570(1.402-1.757)$ & $<0.001^{\mathrm{a}}$ & $1.605(1.433-1.797)$ & $<0.001^{\mathrm{a}}$ \\
\hline Extranodal invasion ( $\geq 2$ vs. $<2$ ) & $1.701(1.490-1.944)$ & $<0.001^{\mathrm{a}}$ & $1.734(1.520-1.981)$ & $<0.001^{\mathrm{a}}$ \\
\hline IPI score (0-2 vs. 3-5) & $1.056(0.931-1.197)$ & 0.398 & - & - \\
\hline Serum LDH level ( $\geq 300$ vs. $<300$ ) & $1.068(0.927-1.230)$ & 0.365 & - & - \\
\hline miR-383-5p expression (High vs. Low) & $0.726(0.649-0.812)$ & $<0.001^{\mathrm{a}}$ & $0.753(0.672-0.842)$ & $<0.001^{\mathrm{a}}$ \\
\hline
\end{tabular}

${ }^{\text {aP }}<0.001$. IPI, International Prognostic Index; LDH, lactate dehydrogenase; miR, microRNA; HR, hazard ratio; CI, confidence interval; -, not available.

and invasion of lymphoma cells was assessed in vitro. The results confirmed that miR-383-5p mimic significantly increased miR-383-5p expression in OCI-LY7 and OCI-LY3 cells $(\mathrm{P}<0.001$; Fig. $3 \mathrm{~A}$ and $\mathrm{B})$.

The results of the CCK- 8 assay demonstrated that the number of OCI-LY7 and OCI-LY3 cells significantly decreased at days 3 and 4 following transfection with miR-383-5p mimic compared with the untreated cells $(\mathrm{P}<0.01$ and $\mathrm{P}<0.001$; Fig. 3C and D). In addition, crystal violet staining demonstrated that overexpression of miR-383-5p significantly inhibited the proliferation of both OCI-LY7 and OCI-LY3 cells $(\mathrm{P}<0.001$; Fig. 3E). The results of the Transwell assay demonstrated that overexpression of miR-383-5p significantly inhibited the invasive ability of both OCI-LY7 and OCI-LY3 cells $(\mathrm{P}<0.001$; Fig. 3F).

miR-383-5p knockdown promotes the proliferation and invasion of DLBCL cells. OCI-LY7 and OCI-LY3 cells were transfected with miR-383-5p inhibitor to decrease miR-383-5p expression, and the results indicated that miR-383-5p inhibitor markedly suppressed miR-383-5p expression in OCI-LY7 and
OCI-LY3 cells $(\mathrm{P}<0.001$; Fig. 4A and $\mathrm{B})$. The results of the CCK-8 assay and crystal violet staining demonstrated that miR-383-5p knockdown significantly promoted the proliferative ability of both OCI-LY7 and OCI-LY3 cells $(\mathrm{P}<0.05$ Fig. 4C-E). Similarly, the results of the Transwell assay demonstrated that miR-383-5p knockdown significantly promoted the invasive ability of OCI-LY7 and OCI-LY3 (P<0.001; Fig. 4F).

\section{Discussion}

Several miRNAs have been demonstrated to play fundamental roles in the initiation and progression of DLBCL. For example, miR-155 knockdown inhibits cell proliferation and facilitates apoptosis of DLBCL in vitro by upregulating SOCS3 expression to suppress JAK-STAT3 signaling (27). In addition, low miR-27b expression is associated with poor overall survival of patients with DLBCL, while high miR-27b expression suppresses the proliferation of DLBCL cells (28).

The results of the present study demonstrated that miR-383-5p expression was downregulated in human DLBCL tissues and related cell lines. In addition, miR-383-5p 
A

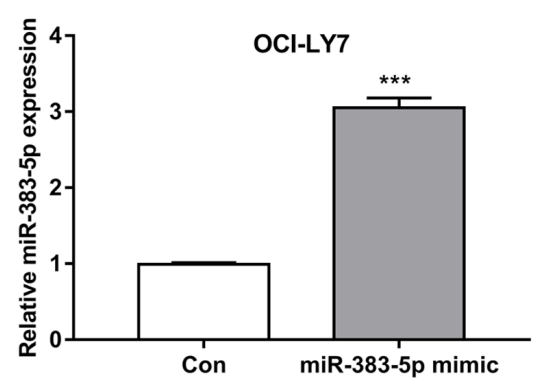

C

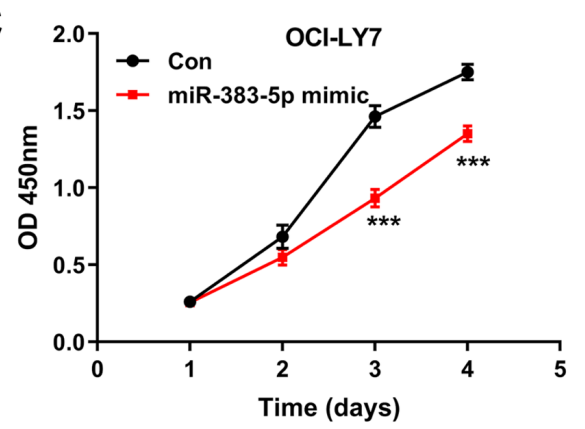

E

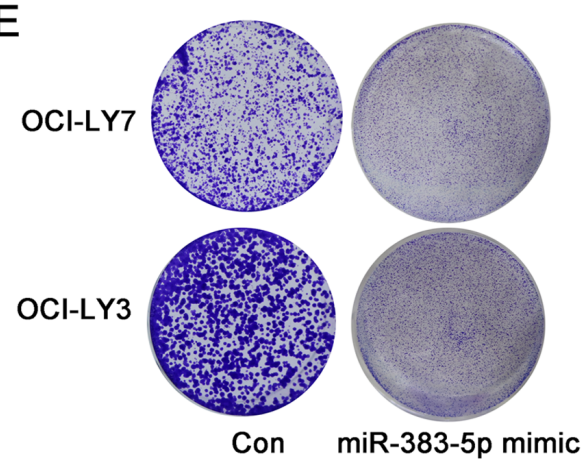

$\mathrm{F}$

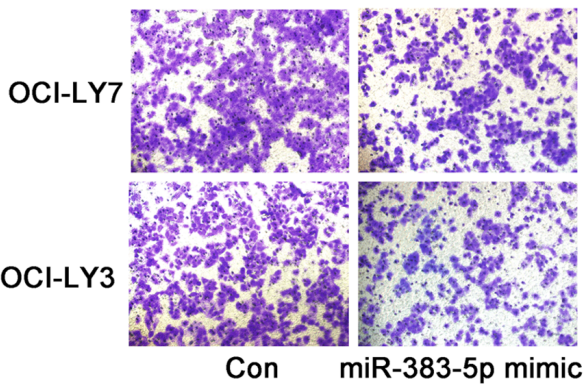

B

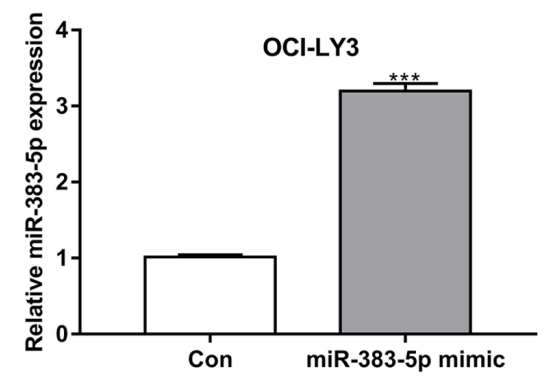

D

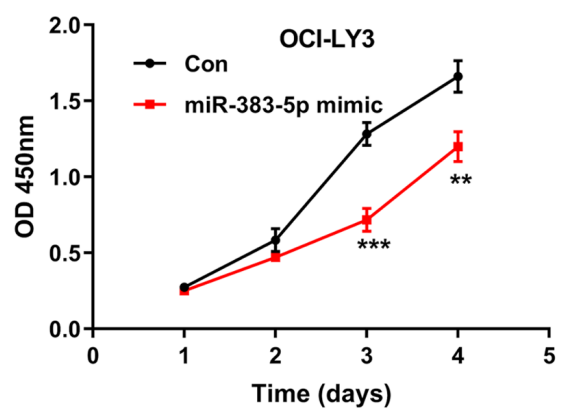

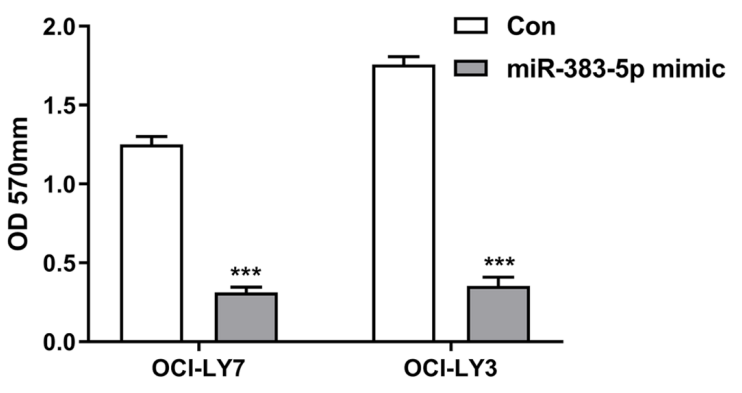

Figure 3. Overexpression of miR-383-5p inhibits the proliferation and invasion of diffuse large B-cell lymphoma cells. miR-383-5p expression was detected in (A) OCI-LY7 and (B) OCI-LY3 cells following transfection with miR-383-5p mimic and negative control, respectively. The Cell Counting Kit-8 assay was performed to assess the effect of miR-383-5p overexpression on the proliferative ability of (C) OCI-LY7 and (D) OCI-LY3 cells. (E) Crystal violet staining was performed to assess the effect of miR-383-5p overexpression on the proliferation of OCI-LY7 and OCI-LY3 cells. (F) The Transwell assay was performed to assess the effect of miR-383-5p overexpression on the invasive ability of OCI-LY7 and OCI-LY3 cells (magnification, x200). Data are presented as the mean \pm standard deviation $(\mathrm{n}=3) .{ }^{* *} \mathrm{P}<0.01,{ }^{* * *} \mathrm{P}<0.001$ vs. Con. miR, microRNA; Con, control; OD, optical density.

expression was closely associated with advanced clinical stage and extranodal invasion. Notably, high miR-383-5p expression predicted favorable clinical prognosis for patients with DLBCL. Taken together, these results suggest that miR-383-5p may serve as an independent prognostic biomarker, as well as a tumor suppressor in patients with DLBCL. Overexpression and knockdown of miR-383-5p were performed in two independent DLBCL cell lines using miR-383-5p mimic and inhibitor, respectively. The effect of altering miR-383-5p expression on the proliferation and invasion of DLBCL cells was assessed. The results demonstrated that overexpression of miR-383-5p significantly inhibited the proliferation and invasion of DLBCL cells, the effects of which were reversed following miR-383-5p knockdown.

The tumor suppressive role of miR-383-5p has been reported in several malignancies, including breast (15), gastric (16) and ovarian cancers (18), as well as lung adenocarcinoma (19). Zhang et al (15) demonstrated that miR-383-5p 
A

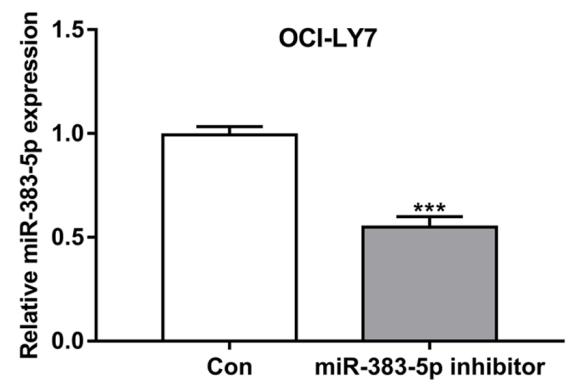

C

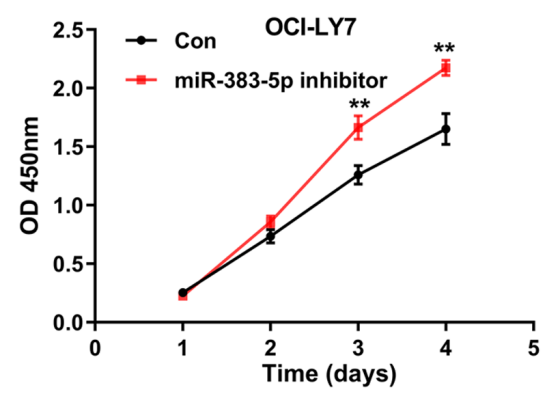

E

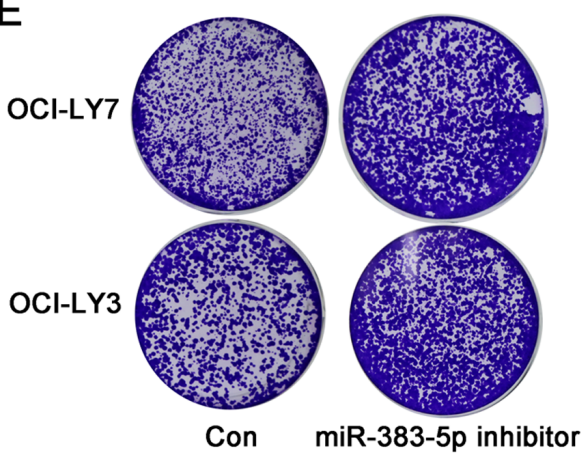

$\mathbf{F}$

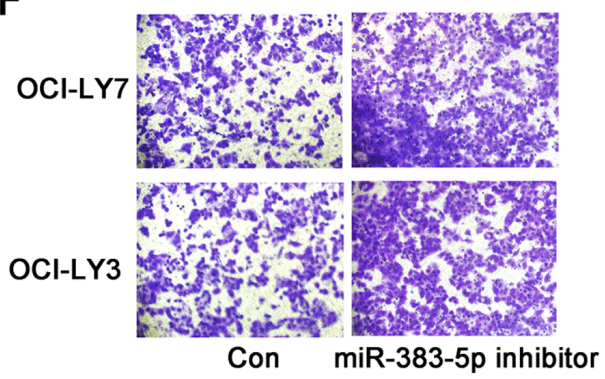

B

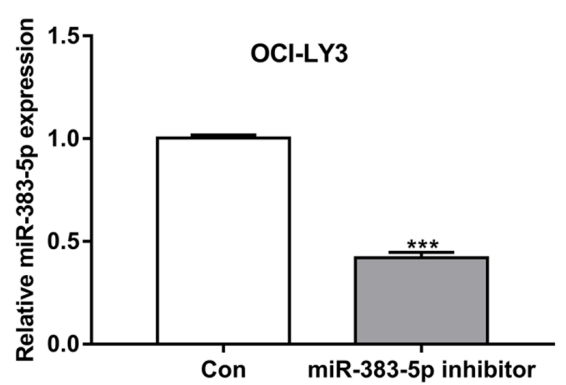

D

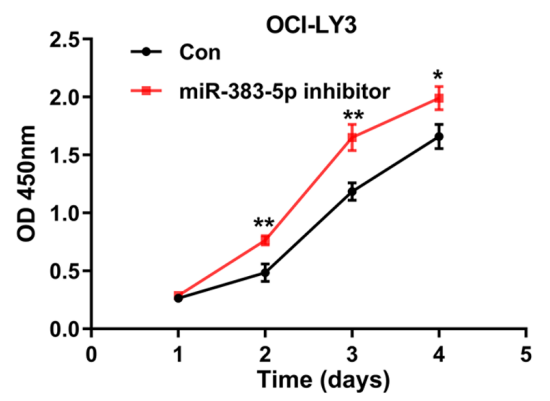

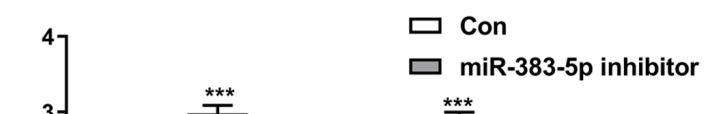
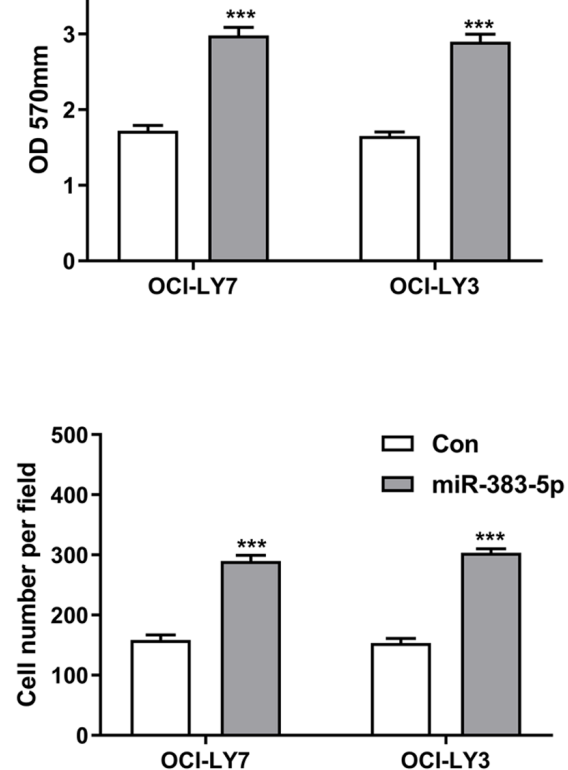

Figure 4. miR-383-5p knockdown promotes the proliferation and invasion of diffuse large B-cell lymphoma cells. miR-383-5p expression was detected in (A) OCI-LY7 and (B) OCI-LY3 cells following transfection with miR-383-5p inhibitor and negative control, respectively. The Cell Counting Kit-8 assay was performed to assess the effect of miR-383-5p knockdown on the proliferative ability of (C) OCI-LY7 and (D) OCI-LY3 cells. (E) Crystal violet staining was performed to assess the effect of miR-383-5p knockdown on the proliferation of OCI-LY7 and OCI-LY3 cells. (F) The Transwell assay was performed to assess the effect of miR-383-5p knockdown on the invasive ability of OCI-LY7 and OCI-LY3 cells (magnification, x200). Data are presented as the mean \pm standard deviation $(\mathrm{n}=3) .{ }^{*} \mathrm{P}<0.05,{ }^{* *} \mathrm{P}<0.01,{ }^{* * *} \mathrm{P}<0.001$ vs. Con. miR, microRNA; Con, control; OD, optical density.

expression is downregulated in breast cancer tissues and respective cell lines. In addition, miR-383-5p can inhibit the proliferation, migration and invasion of breast cancer cells, the findings of which are consistent with the results of the present study. Xu et al (17) reported that overexpression of miR-383-5p suppresses the proliferation and increases the apoptosis of gastric cancer cells, suggesting its prominent role in gastric carcinogenesis. In addition, overexpression of miR-383-5p inhibits cell proliferation, tumor growth and enhances chemosensitivity of ovarian cancer cells by downregulating
TRIM27 expression (18). miR-383-5p expression is downregulated in lung adenocarcinoma tissues, and also exerts antiproliferative effects on lung adenocarcinoma cells (19). Further studies are required to determine the underlying mechanism of miR-383-5p in regulating the progression of DLBCL cells.

In conclusion, the results of the present study demonstrated that miR-383-5p expression is downregulated in DLBCL tissues, and thus may serve as a prognostic biomarker for patients with DLBCL. In addition, miR-383-5p appears to play 
a critical role in inhibiting the proliferation and invasion of DLBCL cells, and thus may act as a putative therapeutic target for effective treatment of DLBCL.

\section{Acknowledgements}

Not applicable.

\section{Funding}

The present study was supported by the Science and Technology Project of Heilongjiang Provincial Department of Education (grant no. 12541372) and the Science and Technology Project of Heilongjiang Provincial Health and Family Planning Commission (grant no. 2013055).

\section{Availability of data and materials}

All data generated or analyzed during the present study are included in this published article.

\section{Authors' contributions}

LYC, BQH and XMZ designed the present study, performed the experiments and analyzed the data. XBY, DDY and LQY collected the clinical samples and analyzed the data. LYC and LQY confirm the authenticity of all the raw data. All authors have read and approved the final manuscript.

\section{Ethics approval and consent for participation}

The present study was approved by the Ethics Committee of the Second Affiliated Hospital of Harbin Medical University (Harbin, China; approval no. ASHHM000152), and performed in accordance with the Declaration of Helsinki. Written informed consent was provided by all patients prior to the study start.

\section{Patient consent for publication}

Not applicable.

\section{Competing interests}

The authors declare that they have no competing interests.

\section{References}

1. Mugnaini EN and Ghosh N: Lymphoma. Prim Care 43: 661-675, 2016.

2. Monfardini S, Zagonel V and Noordijk EM: Lymphomas. Crit Rev Oncol Hematol 27: 157-160, 1998.

3. Xu-Monette ZY, Wu L, Visco C, Tai YC, Tzankov A, Liu WM, Montes-Moreno S, Dybkaer K, Chiu A, Orazi A, et al: Mutational profile and prognostic significance of TP53 in diffuse large B-cell lymphoma patients treated with R-CHOP: Report from an international DLBCL rituximab-CHOP consortium program study. Blood 120: 3986-3996, 2012.

4. Martelli M, Ferreri AJ, Agostinelli C, Di Rocco A Pfreundschuh M and Pileri SA: Diffuse large B-cell lymphoma. Crit Rev Oncol Hematol 87: 146-171, 2013.
5. Jiang $\mathrm{W}$ and Huang $\mathrm{H}$ : The evolution and curative effect of diffuse large B cell lymphoma treatment in China. Zhonghua Xue Ye Xue Za Zhi 35: 357-360, 2014 (In Chinese).

6. Schürch CM, Federmann B, Quintanilla-Martinez L and Fend F: Tumor heterogeneity in lymphomas: A different breed. Pathobiology 85: 130-145, 2018.

7. Matasar MJ and Zelenetz AD: Overview of lymphoma diagnosis and management. Radiol Clin North Am 46: 175-198, vii, 2008.

8. Larrabeiti-Etxebarria A, Lopez-Santillan M, Santos-Zorrozua B, Lopez-Lopez E and Garcia-Orad A: Systematic review of the potential of MicroRNAs in diffuse large B cell lymphoma. Cancers (Basel) 11: 144, 2019.

9. Lee YS and Dutta A: MicroRNAs in cancer. Annu Rev Pathol 4: 199-227, 2009.

10. Wu M, Wang G, Tian W, Deng Y and Xu Y: MiRNA-based therapeutics for lung cancer. Curr Pharm Des 23: 5989-5996, 2018.

11. Du X, Zhang J, Wang J, Lin X and Ding F: Role of miRNA in lung cancer-potential biomarkers and therapies. Curr Pharm Des 23: 5997-6010, 2018.

12. Kabekkodu SP, Shukla V, Varghese VK, D'Souza J, Chakrabarty S and Satyamoorthy K: Clustered miRNAs and their role in biological functions and diseases. Biol Rev Camb Philos Soc 93: 1955-1986, 2018.

13. Lu TX and Rothenberg ME: MicroRNA. J Allergy Clin Immunol 141: 1202-1207, 2018.

14. Vishnoi A and Rani S: MiRNA biogenesis and regulation of diseases: An overview. Methods Mol Biol 1509: 1-10, 2017.

15. Zhang J, Kong X, Shi Q and Zhao B: MicroRNA-383-5p acts as a potential prognostic biomarker and an inhibitor of tumor cell proliferation, migration, and invasion in breast cancer. Cancer Biomark 27: 423-432, 2020.

16. Wei C and Gao JJ: Downregulated miR-383-5p contributes to the proliferation and migration of gastric cancer cells and is associated with poor prognosis. PeerJ 7: e7882, 2019.

17. Xu G, Li N, Zhang Y,Zhang J, Xu R and Wu Y: MicroRNA-383-5p inhibits the progression of gastric carcinoma via targeting HDAC9 expression. Braz J Med Biol Res 52: e8341, 2019.

18. Jiang J, Xie C, Liu Y, Shi Q and Chen Y: Up-regulation of miR-383-5p suppresses proliferation and enhances chemosensitivity in ovarian cancer cells by targeting TRIM27. Biomed Pharmacother 109: 595-601, 2019.

19. Zhao S, Gao X, Zang S, Li Y, Feng X and Yuan X: MicroRNA-383-5p acts as a prognostic marker and inhibitor of cell proliferation in lung adenocarcinoma by cancerous inhibitor of protein phosphatase 2A. Oncol Lett 14: 3573-3579, 2017.

20. Shao B, Fu X, Li X, Li Y and Gan N: RP11-284F21.9 promotes oral squamous cell carcinoma development via the miR-383-5p/MAL2 axis. J Oral Pathol Med 49: 21-29, 2020.

21. Zheng B, Xi Z, Liu R, Yin W, Sui Z, Ren B, Miller H, Gong Q and Liu C: The function of MicroRNAs in B-cell development, lymphoma, and their potential in clinical practice. Front Immunol 9: 936, 2018.

22. Fernando TR, Rodriguez-Malave NI and Rao DS: MicroRNAs in B cell development and malignancy. J Hematol Oncol 5: 7, 2012.

23. de Yébenes VG, Bartolomé-Izquierdo N and Ramiro AR: Regulation of B-cell development and function by microRNAs. Immunol Rev 253: 25-39, 2013.

24. Cheson BD, Fisher RI, Barrington SF, Cavalli F, Schwartz LH, Zucca E, Lister TA; Alliance, Australasian Leukaemia and Lymphoma Group; Eastern Cooperative Oncology Group,et al: Recommendations for initial evaluation, staging, and response assessment of Hodgkin and non-Hodgkin lymphoma: The Lugano classification. J Clin Oncol 32: 3059-3068, 2014.

25. Yang Y, Wang L, Ma Y, Han T and Huang M: The enhanced international prognostic index for diffuse large B-cell lymphoma. Am J Med Sci 353: 459-465, 2017.

26. Livak KJ and Schmittgen TD: Analysis of relative gene expression data using real-time quantitative PCR and the 2(-Delta Delta C(T)) method. Methods 25: 402-408, 2001.

27. Li XD, Li XM, Gu JW and Sun XC: MiR-155 regulates lymphoma cell proliferation and apoptosis through targeting SOCS3/JAK-STAT3 signaling pathway. Eur Rev Med Pharmacol Sci 21: 5153-5159, 2017.

28. Jia YJ, Liu ZB, Wang WG, Sun CB, Wei P, Yang YL, You MJ, Yu BH, Li XQ and Zhou XY: HDAC6 regulates microRNA-27b that suppresses proliferation, promotes apoptosis and target MET in diffuse large B-cell lymphoma. Leukemia 32: 703-711, 2018. 